Research Article

www.jestr.org

\title{
Experimental Control of Curing \& Structural Health Monitoring for Composite Patch Repairs
}

\author{
K.Kalkanis $^{1, *}$, G. J. Tsamasphyros ${ }^{1}$, G. N. Kanderakis ${ }^{1}$, N. Pantelelis ${ }^{2}$, G. Maistros ${ }^{3}$ \\ and A. El. Tsovolos ${ }^{4}$ \\ ${ }^{I}$ The National Technical University of Athens, Faculty of Applied Sciences, Dept. of Mechanics-Lab. of Strength Materials, Zographou \\ Campus, Theocaris Bld., GR-0157 73, Athens, Greece \\ ${ }^{2}$ The National Technical University of Athens, P.O. Box 64078, 15710 Athens, Greece \\ ${ }^{3}$ Inasco Integrated Aerospace Sciences Corporation, 17 Tegeas Str, Argyroupolis 16452, Athens, Greece \\ ${ }^{4}$ University of Portsmouth. Department of Mechanical \& Design Engineering. Anglesea Building Anglesea Rd, Portsmouth, PO1 3DJ \\ Hampshire. UK
}

Received 26 February 2011; Accepted 28 February 2011

\begin{abstract}
Unquestionably structural integrity of composite materials is of great importance and spans from production to service. The presented experimental investigation, supported initially by numerical analysis, addresses the capability of sensing during the curing cycle of Composite Patch Repair (CPR) guaranteeing achievement of optimal cure levels. Results have exhibited identification and completion of cure leading to probable reduction of repair and downtime costs. The sensors were further used post curing for structural integrity where results proved that the optical fibres can render a general state of the strain field inside the patches and possible crack propagation (real time).
\end{abstract}

Keywords: Composite Patch Repair, Cure process optimization, Fibre Bragg Grating Sensors, fatigue loading, Structural health monitoring.

\section{Introduction}

Composite materials are being increasingly used in aerospace applications, due to the advantages they offer. Nevertheless, the need to understand and characterize the modes of failure in these materials and the subsequent quality assurance are mandatory. Low velocity impact loads, eccentrically oriented loading of the structure or manufacturing flaws may lead to delamination between the individual layers of a composite. The generation of delamination in a composite does not necessarily constitute catastrophic failure as it does in common materials. Nevertheless it can lead to points of stress concentration on the layers that carry loads, decrease in stiffness of the structure and/or propagation to a critical surface.

Research has been conducted, proving in general that delamination and shear stresses produced are elevated in such configurations. More specifically, investigated areas include analyzing and finding single lap joint stress distributions either on the adhesive layer or with the assumption of an initial crack in the composite [1,2] and cases of a composite substrate [3].

The use of Fibre Bragg Grating sensors (FBG) that are embedded to composite laminates has proven to be an efficient means of monitoring the strain field of such

*E-mail address: k_kalkanis@hotmail.com ISSN: 1791-2377 (C) 2011 Kavala Institute of Technology. All rights reserved. laminates.

This effort focuses on the use of sensors towards Structural Health Monitoring (SHM) of Composite repair Patches (CPR), which is often used in the aerospace and naval industry. In such applications, the positioning of sensors (especially FBG) is of great interest, given that except from the general strain readings of the composite, they provide the capability of detection, whether a crack has propagated to the metal or a delamination has been created between patch and substrate. The specified sensors can also be used in the curing phase of the composite providing ever so valuable information regarding level of cure and residual strain.

Optical fibre sensors can also be inserted into sensor networks for extensive control [4-6].The scientific effort presents a series of experiments that involve the curing phase of CPR followed by mechanical trials. The curing console uses thermocouples for temperature identification. Further to these, FBG sensors were used. The embedding or optical FBG fibres (3 Bragg gratings as seen in Figure 1) were conducted between first and second ply of the composite patch [7].

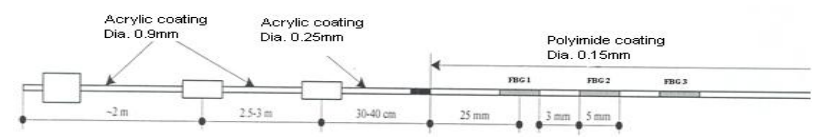

Fig. 1.Sketch allocating sensors along the fibre. 
Measurement data acquisition was conducted by a Micron optics si425 Swept Laser Interrogator. Recording was undertaken by the W3 104 software of Smart Fibres for the entirety of experimental procedures. The results are presented by the order they were conducted, followed by general evaluation.

\section{Cure process}

The curing cycle followed was optimal for the used patches (4 layers of M20 of cytec) with a plateau at $130^{\circ} \mathrm{C}$ for 2 hours with a heating/cooling rate of $3^{\circ} \mathrm{C} / \mathrm{min}$. In Figures $2 \mathrm{a}$ and $b$ snapshots of the experimental procedure are presented. The most crucial problem encountered was the difficulty of data acquisition off the fibre during imposition of vacuum. This fact was due to the pressure being applied onto the fibre through the vacuum bag plies, resulting to data acquisition disruption. The specimen geometry and the exact sensor placement are presented in Figures $3 a$ and $3 b$.

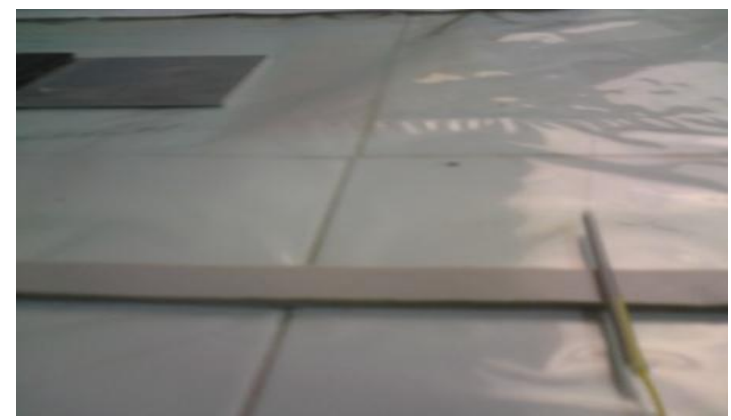

Fig. 2a.The fibre's protective sleeve is placed on the vaccum bag sealant tape during build up.

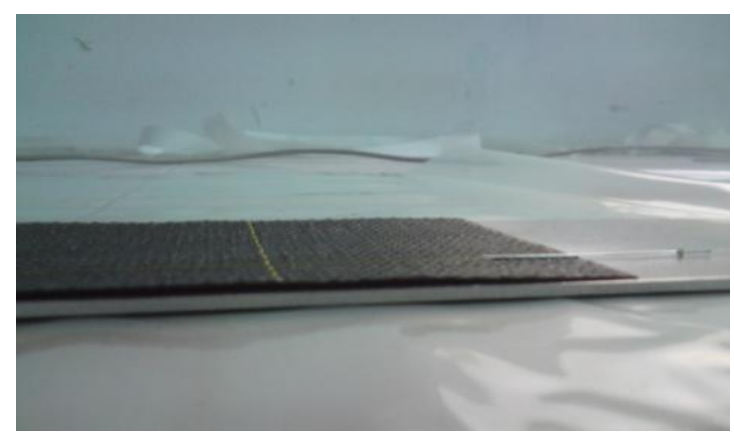

Fig. 2b. Embedding of FBG sensor

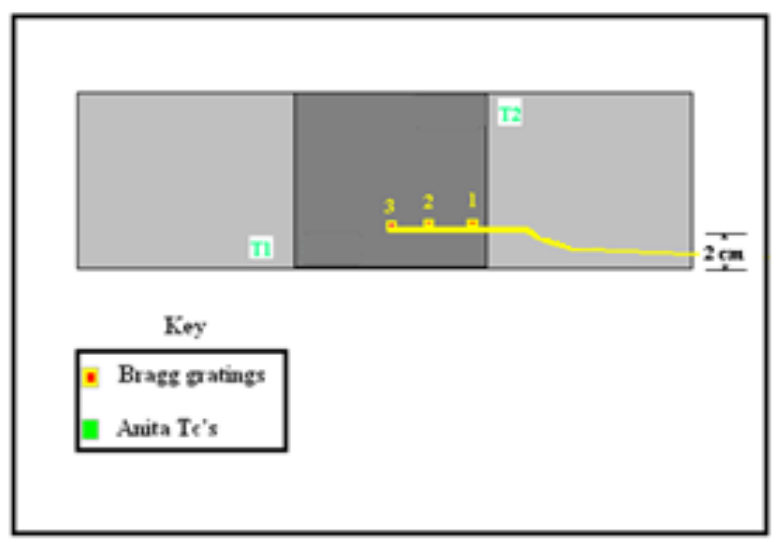

Fig. 3a. Exact sensor placement.

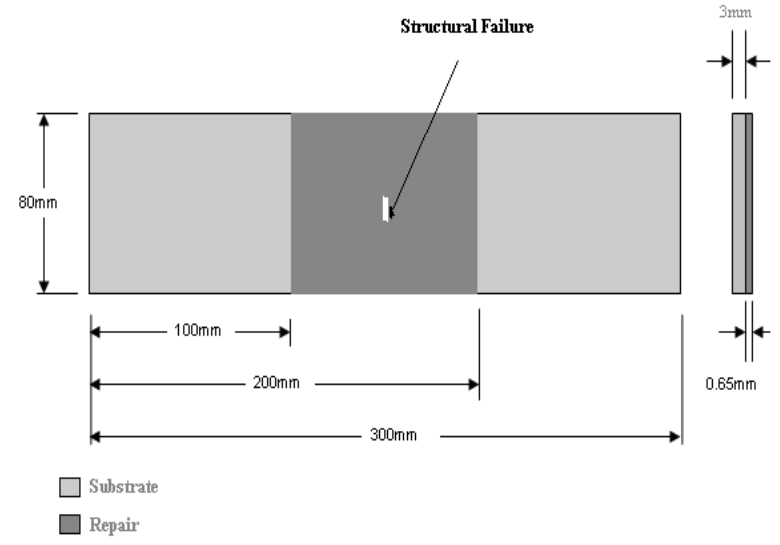

Fig. 3b. Specimen geometry

In Figure 4 the plot of temperature and optical readings are presented over time. For ease of comprehension the following are stated (Table 1):

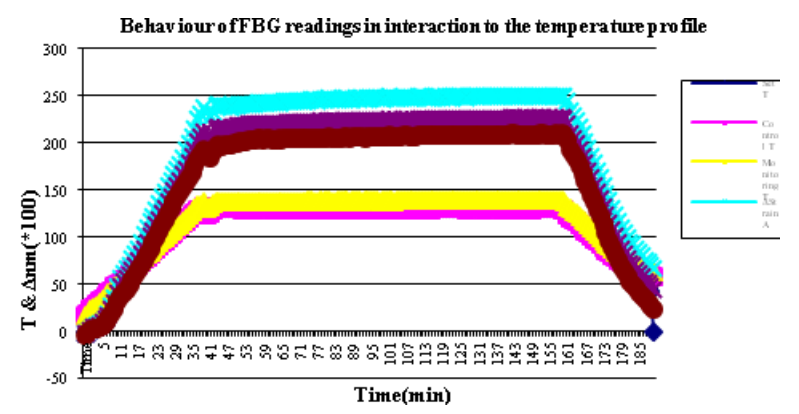

Fig. 4.FBG measurement behaviour.

Table 1. Used symbols and magnitudes

\begin{tabular}{|c|c|}
\hline Symbol & Physical meaning \\
\hline $\mathrm{t}=0$ & Initiation of the curing cycle (Horizontal axis) \\
\hline Set $\mathrm{T}$ & Programmed temperature \\
\hline Control T & $\begin{array}{l}\text { Temperature read off the primary thermocouple } \\
\text { (No 1). }\end{array}$ \\
\hline Monitor T & Temperature read off secondary thermocouples. \\
\hline$\Delta$ strainA & $\begin{array}{l}\text { The change in wavelength for sensor A (nearest to the } \\
\text { crack) in nm, multiplied by } 100 .^{1}\end{array}$ \\
\hline$\Delta$ strainB & $\begin{array}{l}\text { The change in wavelength for sensor B (centrally } \\
\text { placed sensor) in nm, multiplied by } 100 .^{1}\end{array}$ \\
\hline$\Delta$ strainG & $\begin{array}{l}\text { The change in wavelength for sensor } \mathrm{C} \text { (nearest to the } \\
\text { patch boundary) in nm, multiplied by } 100 .{ }^{1}\end{array}$ \\
\hline
\end{tabular}

${ }^{1}$ Multiplication of the wavelength was conducted so as to enable total depiction in a single plot.

Initially, the overall situation is expected. There is a continuous increase in measured $\Delta$ strainduring the plateau. The decrease in FBG optical fibre readings that are recorded at fall of the temperature is steeper compared to the corresponding measurements during temperature rise. Treatment of these measurements [8,9]provides a direct measurement of residual thermal strain.

Strain measurements are in good agreement with the temperature readings. $\Delta$ wavelength readings were computed by subtraction of the corresponding measurements for each sensor at the cure initiation. 
Throughout the experimental procedures certain actions were taken in order to guarantee reliable results. The decrease in temperature at $110{ }^{\circ} \mathrm{C}$ was deemed mandatory so as to avoid premature end of cure. The placement of the primary thermocouple on top of the patch was decided (Figure 5) was conducted even though it will lead to the creation of scarring on the top ply, which is only suited as an experimental practice as it can lead to deterioration of mechanical properties of the finalized repair. Four monitoring thermocouples were added.

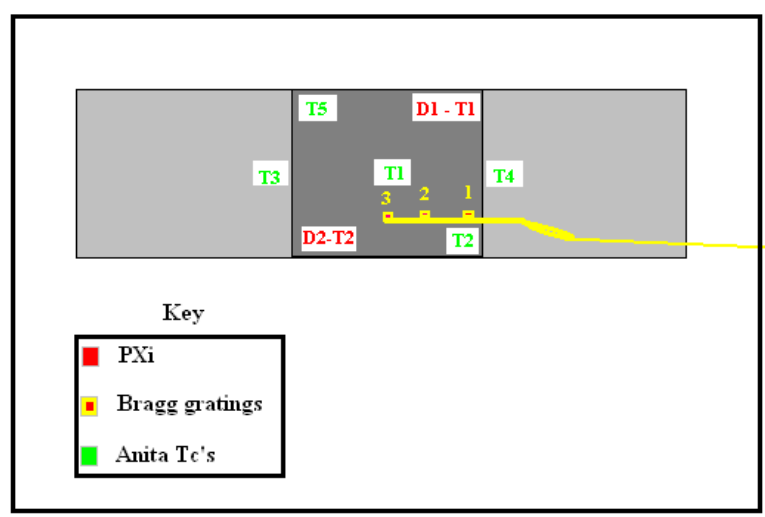

Fig. 5. Sensor placement

In general the following statements can be established: Perfect temperature homogeneity was established, in comparison to primary experiments, as all the readings range $+/-50 \mathrm{C}$ with respect to the programmed temperature. No undesirable increase of the temperature plateau was recorded, as in primary experiments. The above were accomplished due to the placement of the primary thermocouple on the patch and addition of insulation (Figure 6) beneath the specimen. The sensitivity of the overall heating process is consequently validated. The strain measurements ( $\Delta$ strainA, B and $C$ ) agreed to the cure console thermocouples even at the minor fluctuation during the initiation of the plateau. The difference between the heating and cooling slope is identified, which is due to the residual thermal strains post curing. A minor raise in the strain measurements is recorded from the initiation of the plateau (after the temperature fluctuation) up to $t=100 \mathrm{~min}$, a fact that could be due to the curing of the resin.

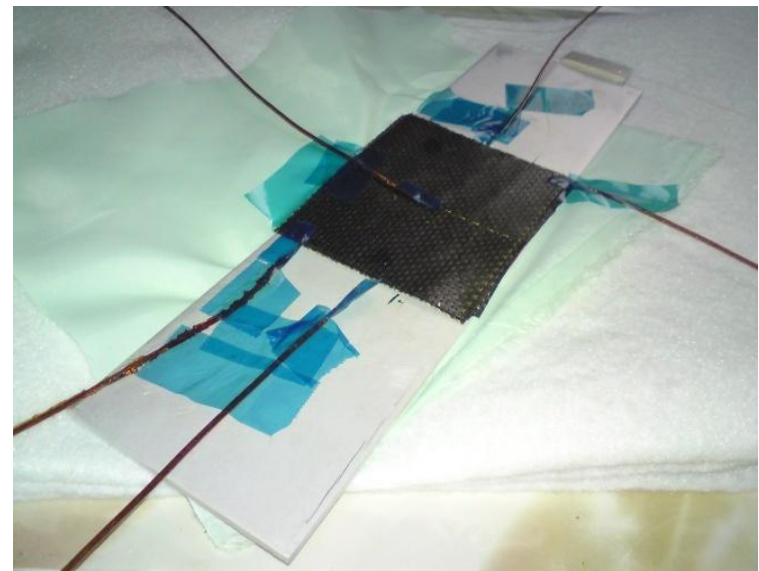

Fig. 6. Addition of Airweave insulating layers below the specimen subject to repair

\subsection{Correction of readings}

The correction of readings involves the use of a second optical fibre, exactly the same with the embedded fibre, was placed near the patch and inside a strain free configuration for accurate decoupling of temperature-strain readings provided by the embedded fibre.

The following points are of importance:

- Compressive deformation is observed at the end of the cycle. Utilizing the FBG factors given by the manufacturer, the computation of thermal residual strain in the patch is possible.

- Increase of strain during the plateau is evident in this experiment, up to $\mathrm{t}=100 \mathrm{~min}$ (i.e. 70 minutes after initiation of the plateau). For ease of comprehension of this observation, a red dotted slope was added (with mean values from $\Delta$ strainC every 10 minutes) which shows that the measurements do not alter at a certain point.

The results are presented in Figure 7.

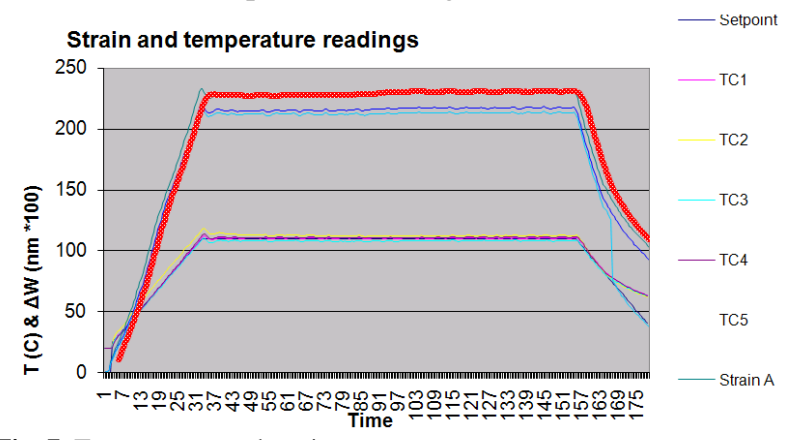

Fig. 7. Temperature and strain measurements

\section{Fatigue loading of FBG enhanced CPR}

Having collected positive results from the use of optic fibres during the cure stage of a composite patch repair, the next step was the online structural health integrity under fatigue loading. Trials were conducted on an Instron 8801 UTM (Figure 8), capable of applying cyclic loading up to 10 tons. The maximum applied stress was equivalent to $45.2 \%$ (193.3 $\mathrm{MPa}$ ) of the substrate's UTS.

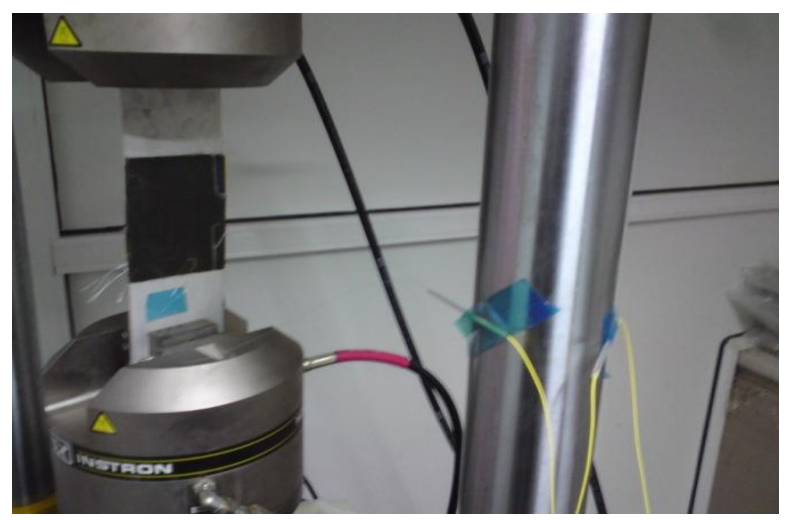

Fig. 8. The fibre part not embedded to the repair was stabilized accordingly and later connected to an si425 Swept Laser Interrogator of Micron optics. 


\subsection{Experimental procedure}

Initially two specimens were subjected to the process in order to familiarize and optimize the experimental procedure. Continuous loading up to the point of failure was decided with simultaneous strain data acquisition off the fibre with a frequency of $0.1 \mathrm{~Hz}$. Figure 9 holds the results where the rise of strains can be observed and a decrease prior to fracture. This phenomenon can be either mechanical or optical.
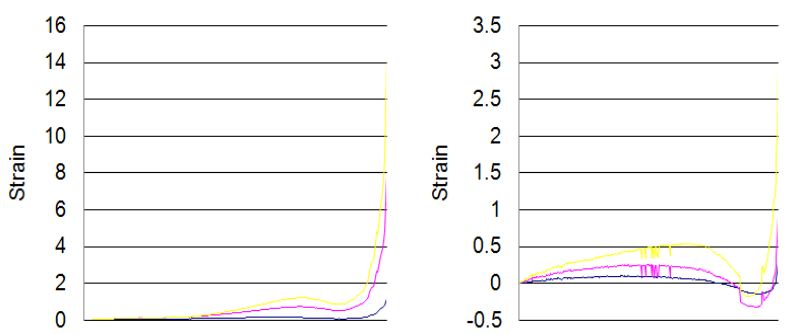

Fig. 9. Preliminary specimens-Results

The repetition of experiments was decided with a different approach. After a certain number of loading cycles $(5000 \mathrm{~N})$, the experiment would come to a halt with the specimen held at tension (mean load). Magnitudes recorded were the length of crack and FBG readings. In this way a direct interrelation between measured strains and failure state can be established.

\subsection{Results}

Results of the initial experiment (Figure 10) show data recording of $4 \mathrm{FBG}$ value sets when the used fibres only possess 3 Bragg gratings. This phenomenon is generated because of the strain field at the third FBG which induces division of the sensor to two separate signals (FBG 3 and FBG 4). The results for a crack length of $15 \mathrm{~mm}$ are of great interest as at that point the crack passes over the fibre's placement axis, a point at which elevated strains are recorded.

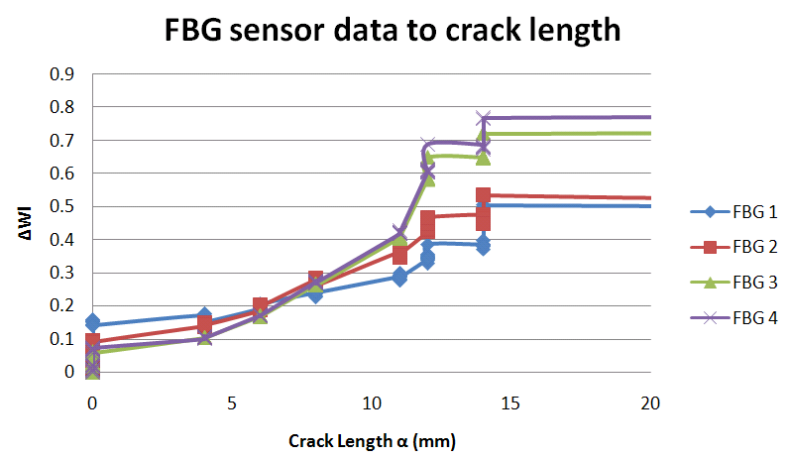

Fig. 10. Results of initial experiment

In the second experiment (Figure11) the data only consist of three sets. Nevertheless, premature fracture with rapid crack propagation is observed. This fact combined with the increase of strain when the crack reached $6 \mathrm{~mm}$ (14mm from the fibre) constitute probable flaw during manufacture-debonding.
FBG sensor data to crack length

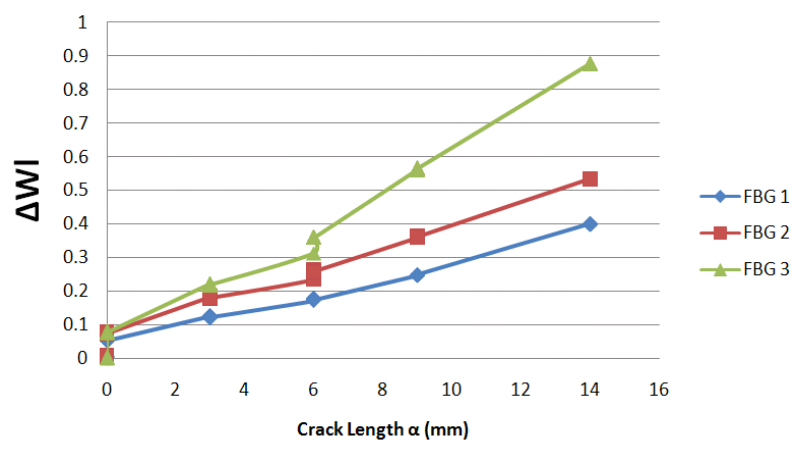

Fig. 11. Results of second experiment.

Furthermore, taking into consideration the statistical mean of specimens with embedded fibres (cycles to fracture) equal to $56.800 \mathrm{~N}$, it is deducted that the incorporation of the specified sensors does not induce problems to the structural integrity (Figure 13) of composite patch repairs at least in low cycle fatigue [8].

The results recorded from the mechanical testing under cyclic loading are presented in Figure 12. The crack halflength was recorded (a) along with the crack initiation up to the point of final fracture of the plate (width $/ 2=40 \mathrm{~mm}$ ). As it can be observed the two repairs exhibited similar performance, finally providing a life extension of $355 \%$ compared to the non repaired structure.

Performance under Fatigue

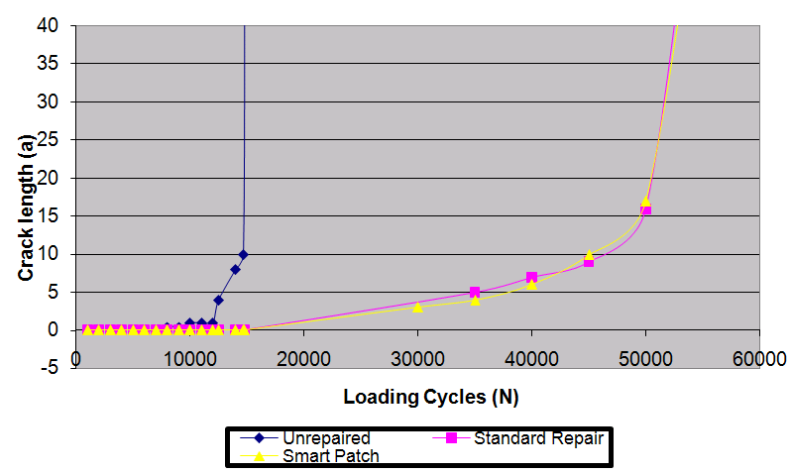

Fig 12. Crack initiation and propagation in relation to the loading cycles.

\section{Conclusions}

Results from this series of experiments are encouraging [10]. Conclusions are presented below:

1. It is possible to localize cure initiation by the proposed sensor setup.

2. The optical sensors present a steep increase in strain measurements

3. It is possible to localize cure completion.

4. The optical sensors reach a plateau.

Curing is completed prior to the end of the temperature plateau (which is recommended). An overall reduction of the curing cycle at an industrial status followed by the certification of quality offered by FBG sensors will lead to important cost reductions.

Results regarding fatigue loading proved that the optical fibres can render a general state of the strain field inside the 
patches and possible crack propagation (real time). Furthermore, it is deducted that the incorporation of the specified sensors does not induce problems to the structural integrity of composite patch repairs in low cycle fatigue.

\section{Acknowledgements}

The optical fibres were manufactured by Professor M. Tur and Dr. Y. Botsev ${ }^{c}$ (Faculty of Engineering, Tel-Aviv University). The help they provided was essential for the presented results.

\section{References}

1. Goland M, Reissner E. The stress in cemented joints. J ApplMech 1944;66: A17-A27.

2. Kairouz KC, Matthews FL. Strength and failure modes of bonded single lap joints between cross-ply adherents. Composites 1993; 24(6):475-84.

3. Wah T. Stress distribution in a bonded anisotropic lap joint.ASME JEngng Mater Technol 1973, 95:174-81.

4. E. Hristoforou, Magnetic Effects in Physical Sensor Design, J. Opt. Adv. Mat., 4, p. 245-260, 2002.

5. E. Maliaritsi, L. Zoumpoulakis, J. Simitzis, P. Vassiliou and E. Hristoforou, Coagulation sensors based on magnetostrictive delay lines for biomedical and chemical engineering applications, Journal of Magnetism and Magnetic Materials, 299, p. 41-52, 2006.

6. Dennison, Christopher R.; Rukosuyev, Maxym V.; Wild, Peter M.; Oshkai, Peter M.; Singlehurst, David A.; Barannyk, Oleksandr L. Local Flow Velocity Measurements Using an In-Fibre Bragg
Grating Sensor, Volume 8, Number 4, pp. 651-658(8).American Scientific Publishers.

7. Raymond Measures. Fiber Opt. Sens. for Comp.Smart Structures, AGARD, CP-531, 1992.

8. Kalkanis K.G. Technological Improvement on Structural Repairs with Composite Patches.NTUA, PhD Thesis. 2009.

9. Crossley S, Marioli-Riga Z, Tsamasphyros G, Kanderakis G, Furnarakis N, Ikiades A, Konstantaki M. Smart Patches: Selfmonitoring composite patches for the repair of aircraft. SPIE's Proc. Int. Symp. on Opt. Tech. for Industrial and Environmental Sensing, Conf. on Intel.Transp. Sensors and Controls, Vol 5272B, Providence, RI, USA, Oct 29-Nov 32003.

10. K.Kalkanis, G. J. Tsamasphyros, G. N. Kanderakis, N. Pantelelis, M. Tur, G. Maistros, Y. Botsev. Experimental Control of Curing via Dielectric \& Fibre Bragg Grating sensors for Composite Patch Repairs. Sensor Letters, (pending publication). American Scientific Publishers. 